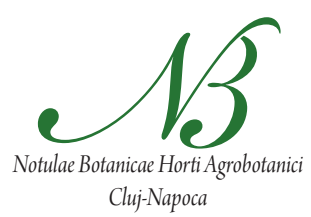

\title{
Morphological and Cytological Response of Selected Recombinant Inbred Lines of Rye (Secale cereale L.) to Nutrient Deprivation Stress Assessed at the Seedling Stage
}

\author{
Miłosz SMOLIK ${ }^{1}$, Patrycja CIELUCH ${ }^{2}$, Kinga MAZURKIEWICZ-ZAPAŁOW ICZ² \\ ${ }^{1}$ West Pomeranian University of Technology in Szczecin, Department of Plant Genetics, Breeding and \\ Biotechnology, Stowackiego 17,71-434Szczecin,Poland:msmolik@zut.edu.pl \\ ${ }^{2}$ West Pomeranian University of Technology in Szczecin, Division of Hydrobiology, Ichthyology \\ of Breeding, Kazimierza Królewicza 4, 71-550 Szczecin, Poland
}

\begin{abstract}
The response of fifty-nine recombinant inbred lines (RILs) of rye $\left(\mathrm{F}_{q}\right)$ to stress caused by low nitrogen and potassium deficiencies in a medium for mature embryo cultures is described in the present study. We demonstrated that the recombinant inbred lines responded in different ways to nutrient stress. On average they developed longer coleoptyles, shorter roots and less numerous roots in the medium with high $\mathrm{N}$ and $\mathrm{K}$ content in comparison with the deficient medium. Also other responses were observed, which included RILs developing significantly shorter roots in the low-nitrogen-potassium treatment when compared to the high-nitrogen-potassium treatment. The lines were clustered depending on their individual response to stress with the use of Ward's agglomerative method. The dendrogram shows four main groups (a-d) with RILs with similar response to caused stress. Susceptible lines were identified in groups 'a'- 'b', while tolerant lines clustered in groups ' $c$ '- 'd'. Cytological analyses of the elongation zone of the seminal roots of seedlings subjected to caused stress were carried out with the use of an inverted microscope. The analysis showed fluorescence of dichlorofluorescein (DCF) in the epidermal zone of the seminal roots, which proves the presence of reactive oxygen species (ROS). The appearance of ROS in the seminal roots could have resulted from the activation of the mechanisms for sensing and responding to induced stress.
\end{abstract}

Keywords: inverted microscopy, nutrient stress, RILs, ROS, rye, visualization

\section{Introduction}

Rye (Secale cereale L.) is a cereal species grown in many countries of Central, Eastern and Northern Europe. This crop presents a wide range of tolerance to edaphic stresses, and like other cereals, it has a characteristic of enhancing its efficiency of nutrient acquisition to overcome the stress of nutrient deficiency or root competition (Geiger and Miedaner, 2009; Rzepka-Plevneš et al., 1997). According to Lynch (2007) and Jia et al. (2008), plant flexibility in biomass allocation, root morphology and root distribution pattern have been found to be an important adaptive mechanism to extract nutrients. It has been shown that a high root:shoot ratio and a specific root length are associated with plants growing in infertile soils (Górny, 1993; Yang et al., 2004).

Identification of the genotypes tolerant to different abiotic stresses with the application of cheap and effective selection methods may facilitate the selection of genotypes useful in breeding programs for low-input agriculture (Oracka and Łapiński, 2006; Rzepka-Plevneš et al., 1999).

In vitro cultures as well as other laboratory methods (e.g. hydroponics, aeroponics or pots) facilitate studying the genetic potential of response of individual genotypes of rye to induced stress (Rzepka-Plevneš et al., 1997; RzepkaPlevneš and Kurek, 1997; Rzepka-Plevneš, 1999), as well as the potential for high-frequency regeneration and induction of somaclonal variability in mature and immature embryo cultures (Rybczyński and Zimny, 1985; RakoczyTrojanowska and Malepszy, 1995). In embryo culture, either the plant develops directly from embryos (RzepkaPlevneš et al., 1997; Rzepka-Plevneš and Kurek, 1997, Rzepka-Plevneš, 1999) or firstly callus is stimulated and then shoots and roots are produced (indirect organogenesisl; Bürün and Poyrazoğlu, 2002; Rakoczy-Trojanowska and Malepszy, 1995). The in vitro selection technique has been used to improve drought tolerance (Gawande et al., 2006; Rzepka-Plevneš et al., 2009), salt tolerance (Yadav et al., 2004), or tolerance to nutrient deprivation in rye (Rzepka-Plevneš et al., 1997; Rzepka-Plevneš and Kurek, 1997; Rzepka-Plevneš, 1999).

Rzepka-Plevneš et al. (1997), Rzepka-Plevneš and Kurek (1997), Rzepka-Plevneš (1999) used cultures of mature embryos to describe the range of variability in different rye genotypes in response to stress caused by nitrogen and potassium deficiencies in a medium, thereby indicat- 
ing the possibility of effective selection of genotypes that differ in the responses to caused stress on the basis of root morphology. It has been demonstrated that the nitrogenpotassium interaction exists at different plant organization levels. In this respect, $\mathrm{K}$ improves the use and utilization of $\mathrm{N}$ and storage of carbohydrates in roots, thus improving NUE (Nutrient-Use Efficiency) (Postma and Lynch, 2011). This interaction is a subject of interest of many studies. $\mathrm{N}$ and $\mathrm{K}$ deficiencies stimulate the activity of antioxidative enzymes, which suggests a role of this stress in reactive oxygen species (ROS) formation. Shin et al. (2005) stated that induced ROS generation under the conditions of nitrogen-potassium deprivation plays a role in triggering gene expression. It has been demonstrated that unlike susceptible genotypes, genotypes tolerant to nutrient stress are able to take up and accumulate macronutrients more effectively (Jian et al., 2008).

Therefore it was the aim of the present study to get selected recombinant inbred lines of rye, described in earlier studies as tolerant and susceptible to $\mathrm{N}$ and $\mathrm{K}$ deficiencies in a medium, into the state of nutrient stress to determine their ability to accumulate selected macroelements in plant dry matter, and to reveal the presence of reactive oxygen species in different preparations of seminal roots with the use of an inverted microscope.

\section{Material and methods}

\section{Plant material}

Plant material included fifty-nine recombinant inbred lines (RILs) $\left(\mathrm{F}_{9}\right)$ of rye (Secale cereale L.) considered as tolerant or susceptible to $\mathrm{N}$ and $\mathrm{K}$ deficiencies in a culture medium. They were derived from the $\mathrm{F}_{2}$ population, obtained by selfing $\mathrm{F}_{1}$ plants obtained as results of crossing two inbred lines 153/79-1 and Ot1-3 with different response to nutrient stress assessed in a mature embryos culture (Rzepka-Plevneš et al., 1997; Rzepka-Plevneš and Kurek, 1997; Rzepka-Plevneš, 1999). The lines were selected for the research on the basis of previous results of morphological, physiological and biochemical analyses of eleven-day-old seedlings of the population of 138 RILs $\left(\mathrm{F}_{7}\right)$ (unpublished results).

\section{Plant mature embryos}

Plant mature embryos culture conditions were established according to Rzepka-Plevneš et al. (1997), RzepkaPlevneš and Kurek (1997), Rzepka-Plevneš (1999). Rye seeds were surface-sterilized by soaking in $1 \%$ solution of $\mathrm{H}_{2} \mathrm{SO}_{4}$ for $15 \mathrm{~min}$ and washed three times with distilled water for $10 \mathrm{~min}$. Then they were soaked in $7 \%$ solution of $\mathrm{NaOCl}$ for $15 \mathrm{~min}$ and washed three times with distilled water for $10 \mathrm{~min}$. Then the seeds were kept in sterile water for $24 \mathrm{~h}$. The mature embryos were prepared with a preparation needle from endosperm and sterilized for $10 \mathrm{~min}$ in $10 \%$ solution of $\mathrm{NaOCl}$, washed three times in sterile water and transferred into proper medium. Four mature em- bryos were placed in each glass tube $(9 \times 3.5 \mathrm{~cm})$ containing $30 \mathrm{ml}$ of the medium. Tubes, closed with aluminium foil and parafilm, were placed for 10 days in a phytotron at $25^{\circ} \mathrm{C}$ under cool white light with 16-hour photope$\operatorname{riod}\left(36 \mu \mathrm{mol} \cdot \mathrm{m}^{-2} \cdot \mathrm{s}^{-1}\right)$ and $75 \% \mathrm{RH}$. The treatments were composed of MS (Murashige and Skoog, 1962) medium. Modified MS medium was used as a control medium and was denoted as high-nitrogen-potassium (HNK). It contained $6.00 \mathrm{mM} \mathrm{N}$ and $2.00 \mathrm{mM} \mathrm{K}$. The test medium, denoted as low-nitrogen-potassium (LNK), was MS medium with reduced content of $\mathrm{N}$ and K-respectively 0.33 $\mathrm{mM} \mathrm{N}$ and $0.33 \mathrm{mM} \mathrm{K}$ (Rzepka-Plevneš, 1999). The $\mathrm{pH}$ of the nutrient mediums was adjusted to 5.7. A completely randomized design was used with replications. Each RIL was represented by 60-80 embryos (15-20 glass tubes), and 30-40 embryos (8-10 glass tubes) were placed in each of the mediums-HNK and LNK, respectively.

\section{Seedlings' traits}

The seedlings were harvested after 11 days when they had one (or two) visible leaves. They were taken from the agar medium and biometric measurements, such as: CLcoleoptyle length $(\mathrm{cm})$, the longest root length-LRL $(\mathrm{cm})$, and root number-RN, were carried out. The experiment was carried out in batches. Eight to ten RILs in every batch in terms of the mentioned parameters were characterized. Apart from normally developed seedlings, also poorly germinating or non-germinated embryos were observed. In the case of contamination the experiment for individual RIL was repeated.

\section{Reactive oxygen species (ROS) visualization and localization}

Reactive oxygen species (ROS) visualization and localization was carried out according to Shin and Schachtman (2004) protocol. The seedlings after eleven-day growth in the HNK and LNK treatments were taken out of the media, washed in distilled water and transferred to Corning tubes $(50 \mathrm{ml})$, to which the liquid HNK and LNK mediums were added. DCFH-DA $\left(2^{\prime}, 7^{\prime}\right.$-dichlorofluorescein diacetate) undergoes deacetylation by intracellular esterase to polar non-fluorescent compound DCFH (2'7' dichlorofluorescein), and then is fast oxidized into highly fluorescent compound DCF (2'7' dichlorofluorescein) as a result of cell activation. In the presence of $\mathrm{H}_{2} \mathrm{O}_{2}$, iron ions and peroxidase, DCF emits a fluorescent signal $\left(\mathrm{A}_{\mathrm{ex}}\right.$ $=498 \mathrm{~nm}, \mathrm{~A}_{\text {emission }}=522 \mathrm{~nm}$ ) with green and greenyellow colour. Corning tubes were transferred back to a phytotron for $30 \mathrm{~h}$. After that time the seedlings were taken out, the seminal roots were washed and placed in a set of newly-prepared tubes containing fresh solutions of the HNK and LNK mediums. From 3 to 4 seedlings among the RILs considered as tolerant and susceptible to nutrient deficiencies in a medium were analysed. Then the following substances were added to the Corning tubes: $30 \mathrm{ml}$ of the HNK medium $+50 \mu \mathrm{M}$ of CM- $\mathrm{H}_{2} \mathrm{DCFDA}$ solution 
284

diluted in dimethyl sulfoxide (DMSO) to the concentration of $0.0025 \%$; $30 \mathrm{ml}$ of the LNK medium $+50 \mu \mathrm{M}$ of CM- $\mathrm{H}_{2} \mathrm{DCFDA}$ solution, and as the positive control-the HNK $+50 \mu \mathrm{M}$ of CM- H $_{2}$ DCFDA solution $+30 \mu \mathrm{l}$ of $\mathrm{H}_{2} \mathrm{O}_{2}$. After 30 minutes the seedling roots were washed with medium solutions not containing CM- $\mathrm{H}_{2}$ DCFDA and observations were made. The elongation zone of the seminal roots of the seedlings growing in the HNK and LNK mediums were subjected to cytological analysis. The pictures were taken with the use of a inverted microscopeNikon (ECLIPSE TE 2000-S) in the laboratory of Division of Hydrobiology; Ichthyology and Biotechnology of Breeding-West Pomeranian University of Technology in Szczecin (Poland).

\section{The total content of macroelements}

The total content of macroelements (N, P, K, Ca, Mg) was assessed according to methods described by Kamińska et al. (1972) and was presented in percentage of seedling's dry matter. The plant material included pooled samples of seedlings of the examined rye recombinant inbred lines considered as tolerant and susceptible to nutrient deficiencies in medium. They were collected separately from the HNK and LNK mediums, respectively after biometric measurements.

\section{Results of biometric measurements}

Results of biometric measurements were statistically analysed with the use of Student's t-test and two-way analysis of variance. Changes in CL, LRL and RN of the seedlings of each analysed RIL were expressed as the value of difference between means of traits assessed under the HNK and LNK treatments. This response was also presented as percentage of the control (HNK), and the traits studied were used as main variables for the clustering of RILs as tolerant and susceptible to nutrient deficiency using Ward's agglomerative method with the use of Statistica 9 software package (StatSoft Poland).

\section{Results}

The recombinant inbred lines of rye significantly differed in terms of response to nitrogen and potassium deficiencies in the medium (Tab. 1). In the HNK treatment they developed coleoptyles on average of the length of $7.04 \mathrm{~cm}$, while in the LNK the coleoptyles were $6.33-\mathrm{cm}$ long. Mean LRL was $4.15 \mathrm{~cm}$ in the HNK treatment, and $4.65 \mathrm{~cm}$ in the LNK treatment. In the HNK and LNK treatments the seedlings of RILs developed on average 3.47 and 3.77 roots, respectively (Tab. 2 ).

A highly significant influence of both genotype, treatment and interaction on the morphological traits of the seedlings was observed (Tab. 1).

The responses of individual RILs among fifty-nine lines subjected to caused stress were statistically significantly different. Some recombinant inbred lines developed more and longer roots in the HNK in comparison with the LNK treatments, or the other way round-shorter and less numerous roots in the HNK medium when compared to the LNK.

The analyzed lines were clustered and characterized according to their response to nutrient stress (Figs. 1,2).

Four separate groups were distinguished on the dendrogram ('a'-d') (Fig. 1). In group 'a'-13 lines were found, in group ' $b$ '-14, 'c'-9, and in ' $d$ '-23 lines. The RILs from each group differed in mean coleoptyle length, root length and root number and their characteristics were presented in fig. 2.

It was found that the analysed seedlings of recombinant inbred lines developed on average shorter coleoptyles in the LNK medium in comparison with the HNK-from $84 \%$ (group 'a') to $98.3 \%$ (group 'c') (Fig. 2A). In group 'a' the seedlings developed the shortest LRL (70\% of the

Tab. 1. Two-way variance analysis of the traits of RILs' seedlings grown in the HNK and LNK treatments

\begin{tabular}{|c|c|c|c|c|c|c|c|c|}
\hline \multirow{2}{*}{ Trait } & \multirow{2}{*}{$\begin{array}{l}\mathrm{N}-\mathrm{K} \\
\text { level }\end{array}$} & \multicolumn{4}{|c|}{ RILs } & \multicolumn{3}{|c|}{$\begin{array}{c}\text { Analysis of } \\
\text { variance }\end{array}$} \\
\hline & & Min & Max & Mean & $s$ & NK & RIL & $\begin{array}{c}(\mathrm{NK} \times \\
\mathrm{RIL})\end{array}$ \\
\hline \multirow{2}{*}{$\begin{array}{c}\mathrm{CL} \\
(\mathrm{cm})\end{array}$} & HNK & 4.24 & 10.48 & 7.04 & 1.31 & ** & ** & $* *$ \\
\hline & LNK & 3.48 & 10.05 & 6.33 & 1.37 & & & \\
\hline \multirow{2}{*}{$\begin{array}{l}\text { LRL } \\
(\mathrm{cm})\end{array}$} & HNK & 1.61 & 8.35 & 4.15 & 1.40 & $* *$ & ** & $* *$ \\
\hline & LNK & 1.72 & 7.29 & 4.61 & 1.69 & & & \\
\hline \multirow{2}{*}{$\mathrm{RN}$} & HNK & 2.38 & 5.09 & 3.47 & 0.62 & $* *$ & $* *$ & ** \\
\hline & LNK & 1.76 & 6.16 & 3.77 & 0.88 & & & \\
\hline
\end{tabular}

ns-Not significant; ${ }^{*} \mathrm{P}<0.05,{ }^{* *} \mathrm{P}<0.01$ significant differences between RILs

Tab. 2. Two-way analysis of variance for total content of macronutrients in percentage of dry matter of the leaves of the seedlings grown in the HNK and LNK treatments

\begin{tabular}{|c|c|c|c|c|}
\hline & \multicolumn{4}{|c|}{ Group of RILs } \\
\hline & & TOL & SUSC & \\
\hline \multirow[t]{3}{*}{$\mathrm{N}$} & HNK & 4.20 & 4.47 & $0.73^{* *}$ \\
\hline & LNK & 4.13 & 2.93 & ns \\
\hline & & $2.07^{* *}$ & $1.54^{* *}$ & \\
\hline \multirow[t]{3}{*}{$\mathrm{P}$} & HNK & 0.96 & 0.83 & ns \\
\hline & LNK & 0.87 & 0.75 & ns \\
\hline & & $\mathrm{ns}$ & ns & \\
\hline \multirow[t]{3}{*}{$\mathrm{K}$} & HNK & 2.93 & 2.81 & ns \\
\hline & LNK & 2.30 & 1.26 & $1.04^{* *}$ \\
\hline & & $0.63^{* *}$ & $1.55^{* *}$ & \\
\hline \multirow[t]{3}{*}{$\mathrm{Ca}$} & HNK & 0.13 & 0.13 & ns \\
\hline & LNK & 0.16 & 0.21 & ns \\
\hline & & $\mathrm{ns}$ & ns & \\
\hline \multirow[t]{2}{*}{$\mathrm{Mg}$} & HNK & 0.12 & 0.12 & ns \\
\hline & LNK & 0.12 & 0.13 & ns \\
\hline
\end{tabular}

TOL-tolerant; SUSC-susceptible; ns-not significant; ${ }^{*} \mathrm{P}<0.05,{ }^{* *} \mathrm{P}<0.01$ significant differences between RILs 


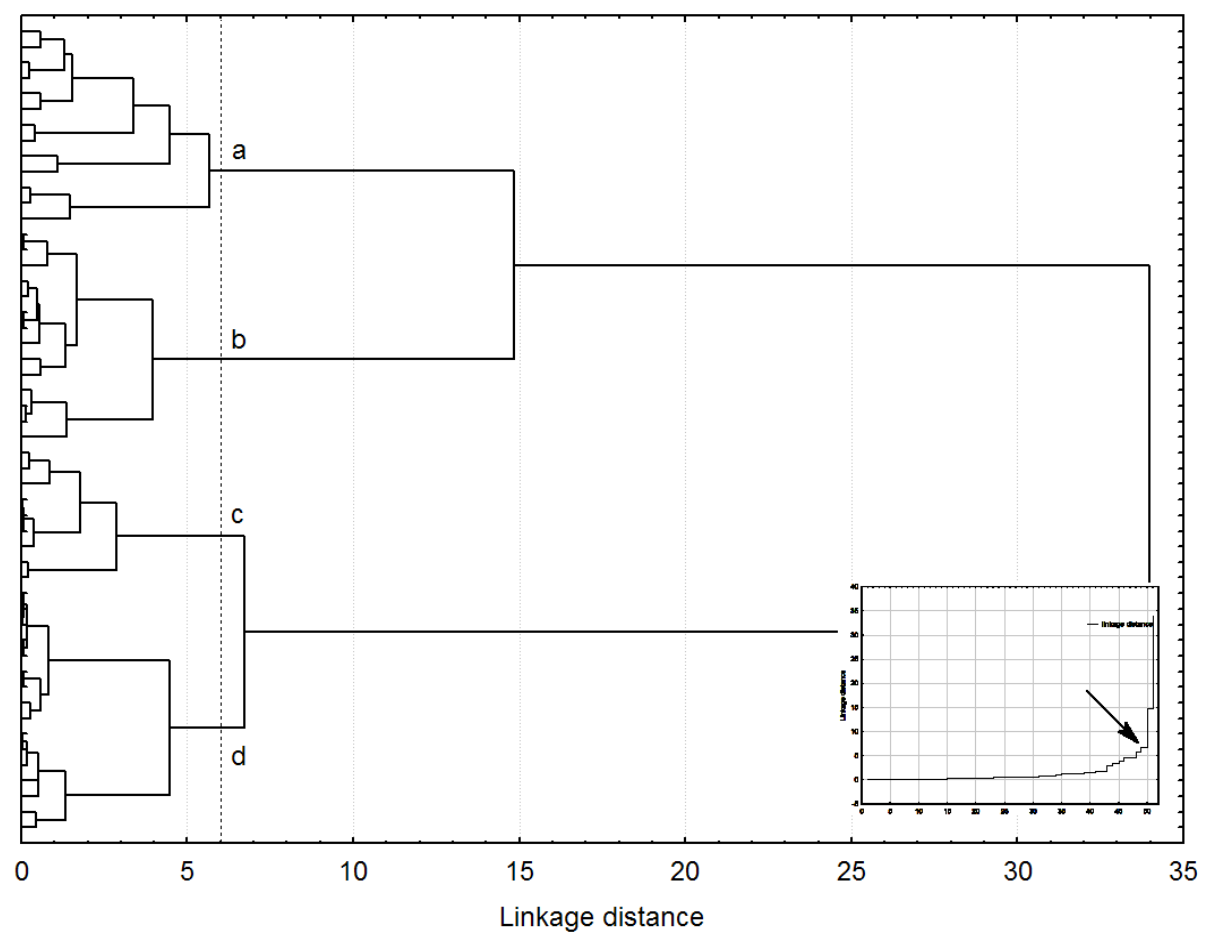

Fig. 1. Clustering of RILs $\left(\mathrm{F}_{q}\right)$ of rye. The vertical lines indicate the cut-offs used to form the groups (a-d) according to the presented scree-plot
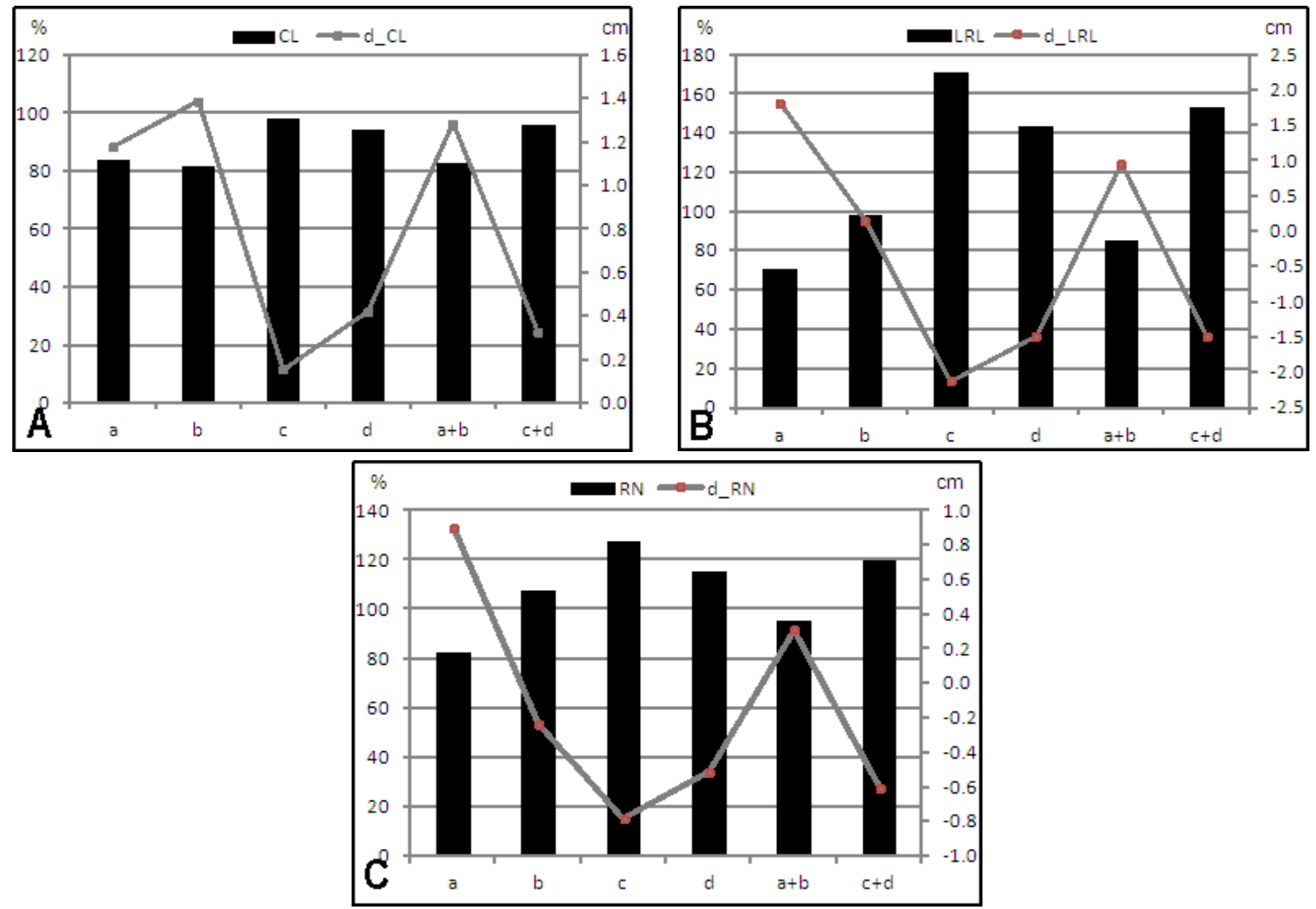

Fig. 2. The RILs' response to induced stress presented as percentage of the control (the HNK) and additionally as the value of difference (d_) between means of each trait and for each group of RILs ('a'-'d') grown in the HNK n comparison with the LNK treatments, as presented in Fig. 1. Groups ' $a$ ' ' $b$ ' and 'c'+'d' were presented as results of simulation of the morphological variability of RILs combined from groups 'a'- 'b' and 'c'-'d', respectively. A. CL-coleoptyle length, B. LRL-the longest root length, C. RN-root number 

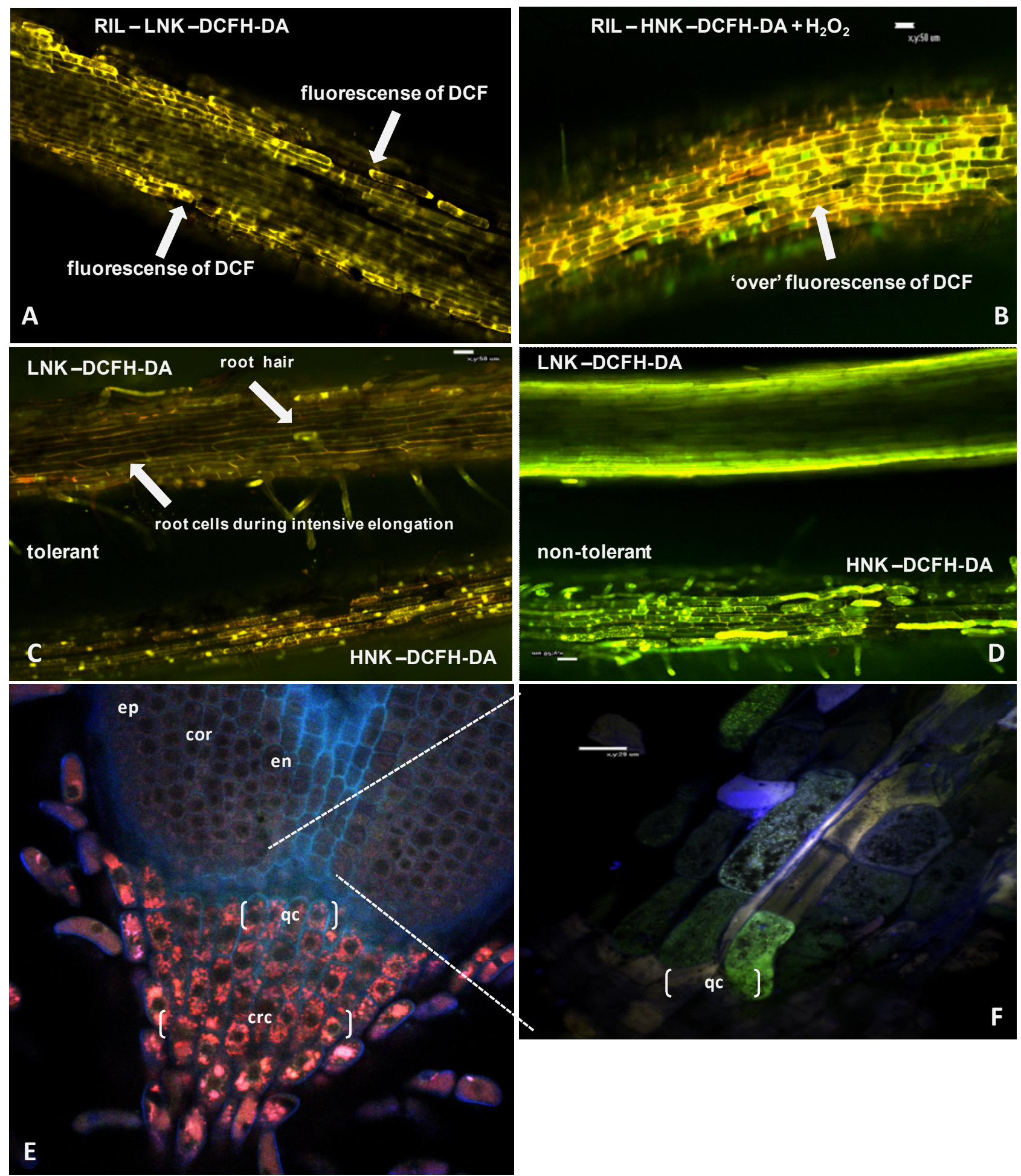

Fig. 1. Confocal images of the seminal roots of the selected RILs of rye described as tolerant and susceptible to nutrient stress caused by $\mathrm{N}$ and $\mathrm{K}$ deficiencies, grown in different treatments (HNK and LNK) under in vitro conditions. Scale bars $=50 \mu \mathrm{m}$.

control), in group 'b'-the trait value amounted to $98.6 \%$ of the control, in groups ' $c$ ' and 'd'-171 and $143.8 \%$, respectively (Fig. 2B). Similarly as in the case of LRL, the RILs clustered in group 'a' developed the smallest number of seminal roots (on average $82 \%$ of the control), in group 'b'-107.6\% of the control, in 'c'-127.6\%, while in group 'd'$115.5 \%$ (Fig. 2C).

Positive correlations were found for morphological traits of RILs between CL and LRL $(\mathrm{r}=0.64, \mathrm{p}<0.01 ; \mathrm{r}$ $=0.49, \mathrm{p}<0.05), \mathrm{CL}$ and $\mathrm{RN}(\mathrm{r}=0.43 ; \mathrm{r}=0.43, \mathrm{p}<0.05)$ 
and between LRL and RN $(r=0.53, p<0.05 ; \mathrm{r}=0.65$, $\mathrm{p}<0.01)$ in the HNK and LNK treatments, respectively.

The conducted research revealed that more total $\mathrm{N}$ in the HNK medium was noted in the pooled sample of tolerant lines in comparison with susceptible lines (Tab. 2). The difference was highly significant. Both groups of seedlings accumulated significantly less nitrogen in the dry matter of coleoptyles in the LNK medium when compared to the HNK medium, and the group of susceptible lines accumulated less nitrogen in comparison with the tolerant group. The content of $\mathrm{P}, \mathrm{Mg}$ and $\mathrm{Ca}$ in the dry matter of coleoptyles of the both groups of RILs did not differ significantly between the groups as well between the treatments. Significantly less K was found for both groups in the dry matter of coleoptyles of the seedlings grown in the LNK treatment in comparison with the HNK, and in the LNK treatment significantly more $\mathrm{K}$ was accumulated by the coleoptyles of the tolerant lines (Tab. 2).

\section{ROS visualization}

Selected cytological preparations of the elongation zone of seminal roots (Photo 1 A-D) and apical meristems (Photo 1E-F) were analysed. More intense fluorescence of dichlorofluorescein (DCF) was observed in the epidermal zone of seminal roots in comparison with the cortex/endodermis zone (Photo 1A). The control over-fluorescence of dichlorofluorescein (DCF) was observed in the whole preparation after adding $30 \mu \mathrm{l}$ of $\mathrm{H}_{2} \mathrm{O}_{2}$ to the liquid medium (Photo 1B). In the tolerant lines, lower fluorescence of dichlorofluorescein was noted in the elongation zone of seminal roots in the LNK medium when compared to the HNK treatment (Photo 1C), while in the susceptible lines fluorescence in the LNK treatment was more intense in comparison with the HNK (Photo 1D).

It should be emphasized that the conclusions were drawn only from 2-3 seedlings of different RILs randomly selected among the group of tolerant and susceptible plants, respectively, while the intensity of fluorescence of dichlorofluorescein (DCF) in the roots growing in the HNK or LNK medium was visually assessed, not referred to specific units. The morphology of the apical meristem of a rye seedling was presented in Photo 1E-F.

\section{Discussion}

The results of the present study confirm a wide range of variability in response of rye recombinant inbred lines to nutrient deficiencies. The physiological mechanism of the growth of plant seedlings in HNK is well known and conditioned among other things by the operation of different mechanisms for sensing and signalling of the presence and uptaking of $\mathrm{NO}_{3}^{-}$ions by roots (Zhang and Forde, 1998) as well as due to the feedback mechanisms decreasing the expression of nitrate transporter reductase (NRT2) genes in a way regulating the influx of $\mathrm{NO}_{3}^{-}$ions into a plant (Leyal et al., 1999). The plant response to nutrient defi- ciencies is also typical. However, it is a very complicated cellular mechanism responding to changes in the availability of macronutrients (including nitrogen and potassium) in the root environment and in the ability to uptake and accumulate these elements (Chun et al., 2005; Łapiński, 2006; Oracka and Ruffel et al., 2011).

Under the conditions of $\mathrm{N}$ and $\mathrm{K}$ deficiencies, typical responses of rye seedlings were observed, i.e. shorter coleoptyles, of the same length or longer and more numerous seminal roots in comparison with the seedlings growing in the HNK medium. Similar results were presented by Rzepka-Plevneš et al. (1997), Rzepka-Plevneš and Kurek (1997), Rzepka-Plevneš (1999), Rzepka-Plevneš and Kulpa (1999) for mature embryos of rye of open-pollinated cultivars, breeding strains or selected populations, examined in in vitro cultures, in comparison with the range of variability of CL, LRL or RN. Górny (1993), Górny and Szołkowska (1996) observed similar responses of barley and oats to $\mathrm{N}$ and $\mathrm{P}$ deficiencies in hydroponics, and Mi et al. (2005) in maize. According to Chun et al. (2005) and Guo et al. (2005), shorter and less numerous roots of the seedlings of the selected RILs of rye in the LNK medium when compared to the HNK medium may depend on genotype and result from plant sensing of the level of available nitrogen forms as extremely low.

Høgh-Jensen and Pedersen (2003) found that low K levels may affect the root morphology in different species: barley, rye, perennial ryegrass or lucerne. However, crops modify their root hair length in response to low $\mathrm{K}$, and thereby maintain the uptake from sparingly soluble $\mathrm{K}$ sources (Jia et al., 2008).

In the root elongation zone of Arabidopsis, Shin et al. (2005) showed that ROS increase and their localization differs according to the specific nutrient of which roots are deprived. ROS also play a role in the regulation of gene expression in response to the deficiency of several macronutrients, including potassium, nitrogen and phosphorus and other edaphic stresses (Esfandiari et al., 2011).

The ROS accumulation in potassium- and nitrogendeprived roots was localized more intensely in the epidermis than in the cortex, while in the situation of root deprivation of phosphorus they were accumulated more intensely in the cortical zone (Shin et al., 2005). According to Shin et al. (2005), changes in ROS concentrations may suggest that root hair cells are important for response to nitrogen and potassium deprivation and the operation of the mechanisms sensing the state of deficiency of both $\mathrm{N}$ and $\mathrm{K}$.

Fluorescence of DCF (dichlorofluorescein) obtained for the selected RILs of rye with the use of an confocal microscope was localized as described for Arabidopsis by Shin et al. (2005) in the epidermal zone of eleven-day-old rye roots. This fact may indicate that the test used for the evaluation of the response of RILs of rye to nutrient stress was able to express their genetic differences (Rzepka-Plevneš $e t$ al., 1997; Rzepka-Plevneš and and Kurek, 1997). 
288

Both in the HNK and LNK medium the seedlings of the lines considered as tolerant accumulated more total nitrogen in dry mass of coleoptiles in comparison with the seedlings considered as susceptible. Unlike in the LNK treatment, in the HNK treatment the differences turned out to be significant. Similar differences were obtained for $\mathrm{K}$. The tolerant lines absorbed more $\mathrm{K}$ from the LNK medium when compared to the susceptible lines. While the differences in the case of the HNK medium were not significant, they were highly significant in the case of the LNK medium. It should be emphasized that the assessment of macroelements was carried out in pooled samples; therefore the presented results should be treated as preliminary.

Jia et al. (2008) reported that the roots of a highly efficient genotype had a greater ability to tolerate low K stress and absorb $\mathrm{K}$ from a deficient medium. At low $\mathrm{K}$ level, the length, surface area, volume, and count of the roots of K-efficient genotypes were higher. It indicates that the genotypic difference for $\mathrm{K}$ efficiency in rice had a close relationship with root morphology (Jia et al., 2008). According to the above authors, the results for rice are in accordance with those for ryegrass (Mengel and Steffens, 1985) or wheat (Zou et al., 2001). Oracka and Łapiński (2006) described $N$ and P uptake and utilization efficiency in two sets of substitution lines of hexaploid triticale and concluded that the differences were dependent on the homology group of a substitution and varietal background of the triticale genotype. They demonstrated that the $\mathrm{R}$ genome causes high uptake of the nutrients, and the $\mathrm{D}$ genome enables high efficiency of their metabolism .

\section{Conclusions}

The results of the present experiment confirmed different responses of the examined lines of rye to stress caused by $\mathrm{N}$ and $\mathrm{K}$ deficiencies in a medium, described in previous studies. The Ward's dendrogram shows lines extreme differ in the response to stress caused by $\mathrm{N}$ and $\mathrm{K}$ deficiencies in a medium, clustered in the groups ' $a$ '- ' $b$ ' and ' $c$ '- 'd'. It was possible to select lines with different response to stress within the examined population of fifty-nine analysed RILs on the basis of previous research. According to literature data, the greater intensity of fluorescence of DCF in the epidermis than in the cortex/endodermis zone of deprived roots confirms the usefulness of assessed in vitro tests for describing individual responses of rye seedlings to nutrient stress caused by nitrogen and potassium deficiencies. This was also confirmed by demonstration of the ability of the seedlings of tolerant RILs to accumulate a greater amount of $\mathrm{N}$ and $\mathrm{K}$ in dry matter in comparison with the susceptible lines. The results of the present study confirm the opinion of many authors on the usefulness of laboratory tests for description of variability in terms of plant response to nutrient stress and selection of desired genotypes.

\section{Acknowledgements}

This work was supported by the Polish Ministry of Science and Higher Education under grant No. N N302 281936.

\section{References}

Bürün B, Poyrazoğlu EÇ (2002). Embryo culture in barley (Hordeum vulgare L.). Turk J Bot 26:175-180.

Chun L, Mi GH, Li JS, Chen FJ, Zhang FS (2005). Genetic analysis of maize root characteristics in response to low nitrogen stress. Plant Soil 276:369-382.

Esfandiari E, Enayati V, Abbasi A (2011). Biochemical and physiological changes in response to salinity in two durum wheat (Triticum turgidum L.) genotypes. Not Bot Hort Agrobo 39(1):165-170.

Gawande ND, Mahurkar DG, Rathod TH, Jahagidar SW and Shinde SM (2005). In vitro screening of wheat genotypes for drought tolerance. Annals Plant Physiol 19:162-168.

Geiger HH, Miedaner T (2009). Rye breeding, in Cereals, Carena MJ (Ed.). Springer-Science and Business Media, LLC 157-181.

Górny AG (1993). Differences in root and shoot response to limited $\mathrm{N}$-supply in oat and spring barley. J Agron Crop Sci 171:161-167.

Górny AG, Szołkowska A (1996). Effects of selection for more vigorous seminal roots in two cross populations of oat (Avena sativa L.). J Appl Genet 37(4):331-344.

Guo Y, Mi GH, Chen F, Zhang F (2005). Effect of $\mathrm{NO}_{3}^{-}$supply on lateral root growth in maize plants. J Plant Physiol Mol Biol 31:90-96.

Høgh-Jensen H, Pedersen MB (2003). Morphological plasticity by crop plants and their potassium use efficiency. J Plant Nutr 26:969-984.

Jia Y, Yang X, Feng Y, Jilani G (2008). Differential response of root morphology to potassium deficient stress among rice genotypes varying in potassium efficiency. J Zhejiang Univ Sci B 9(5):427-434.

Kamińska W, Kardasz T, Strahl A, Bałucka T, Walczak K, Filipek P (1972). The methods of analysis in the agricultural chemical stations. Part II. Plant analysis (in Polish). IUNG, Puławy.

Lejay L, Tillard P, Lepetit M, Olive FD, Filleur S, Daniel-Vedele F, Gojon A (1999). Molecular and functional regulation of two $\mathrm{NO}_{3}$ - uptake systems by $\mathrm{N}$ - and $\mathrm{C}$-status of Arabidopsis plants. Plant J 18:509-519.

Lynch JP (2007). Roots of the second green revolution. Aust J Bot 55:493-512.

Mengel K, Steffens D (1985). Potassium uptake of rye-grass (Lolium perenne) and red clover (Trifolium pratense) as re- 
lated to root parameters. Biol Fert Soils 1:53-58.

Mi GH, Chen FJ, Zhang FS (2007). Physiological and genetic mechanisms for nitrogen use efficiency in maize. J Crop Sci Biotech 10:57-63.

Murashige T, Skoog F (1962). A revised medium for rapid growth and bioassays with tabacco tissue clusters. Plant Physiol 15:473-497.

Oracka T, Łapiński B (2006). Nitrogen and phosphorus uptake and utilization efficiency in $\mathrm{D}(\mathrm{R})$ substitution lines of hexaploid triticale. Plant Breed 125: 221-224.

Postma JA, Lynch JP (2011). Root cortical Aerenchyma enhances the growth of maize on soils with suboptimal availability of nitrogen, phosphorus, and potassium. Plant Physiol 156:1190-1201.

Rakoczy-Trojanowska M, Malepszy S (1995). Genetic factors influencing regeneration ability in rye (Secale cereale L.). II. Immature embryos. Euphytica 83:233-239.

Ruffel S, Krouk G, Ristova D, Shasha D, Birnbaum KD, Coruzzi GM (2011). Nitrogen economics of root foraging: Transitive closure of the nitrate-cytokinin relay and distinct systemic signaling for $\mathrm{N}$ supply vs. demand. PNAS 108(45):18524-18529.

Rybczyński JJ, Zimny J (1985). Screening of cultivar varieties of Secale cereale L. in order to obtain somatic embryogenesis. Proc Eucarpia Meeting of the Cereal Section on Rye, Svalov, June 11-13, 33-53.

Rzepka-Plevneš D (1999) Variability of tolerance to nitrogen and potasium deficiencies in original $\left(\mathrm{S}_{0}\right)$ and selected $\left(\mathrm{S}_{1}-\right.$ $\mathrm{S}_{2}$ ) rye populations, assessed during in vitro cultures. Plant Breed Seed Sci 43(1):47-63.

Rzepka-Plevneš D, Krupa-Małkiewicz M, Kurek J, Smolik M (2009). Effects of water deficits on development and yield of rye varieties differing in tolerance at seedling stage. J Food Agric and Environ 7(3-4):492-495.
Rzepka-Plevneš D, Kulpa D (1999). Agronomic properties of rye populations selected for tolerance to nutrition deficiency under laboratory conditions (in Polish). Biul IHAR 211:259-265.

Rzepka-Plevneš D, Kurek J (1997). The tolerance of some rye varieties and breeding strains to nutrition deficiency using in vitro cultures. Plant Breed Seed Sci 41:41-49.

Rzepka-Plevneš D, Marciniak H, Śmiech M (1997). The evaluation of rye (S. cereale L.) inbred lines tolerance to nutrition deficiency by in vitro test (in Polish). Biul IHAR 203:137146.

Shin R, Berg RH, Schachtman DP (2005). Reactive oxygen species and root hairs in Arabidopsis root response to nitrogen, phosphorus and potassium deficiency. Plant Cell Physiol 46(8):1350-1357.

Shin R, Schachtman DP (2004). Hydrogen peroxide mediates plant root cell response to nutrient deprivation. PNAS 101:8827-8832.

Yadav N, Uppal S, Sehrawat AR and Singh KP (2004). In vitro callus growth, selection of $\mathrm{NaCl}$ tolerant cell lines and plant regeneration in wheat. Nat J Plant Improv 6:130-131.

Yang XE, Liu JX, Wang WM (2004). Potassium internal use efficiency relative to growth vigor, potassium distribution, and carbohydrate allocation in rice genotypes. J Plant Nutr 27 (5):837-852.

Zhang H, Forde BG (1998) An Arabidopsis MADS box gene that controls nutrient induced changes in root architecture. Science 279:407-409. 